\title{
Single Molecule Imaging of a $\pi$-Conjugated Polymer in Tetrahydrofuran Solution at Room Temperature
}

\author{
Ken-ichi Shinohara, $*, *,+$ Shingo YamaguCHI, ${ }^{* *}$ and Hideo HiguCHI $*, * *$ \\ ${ }^{*}$ Center for Interdisciplinary Research (CIR), Tohoku University, Aramaki-aza-aoba, Sendai 980-8578, Japan \\ ** Department of Metallurgy, Graduate School of Engineering, Tohoku University, Aoba-yama 02, Sendai 980-8579, Japan
}

(Received May 25, 2000; Accepted July 12, 2000)

KEY WORDS Single Molecule Detection / $\pi$-Conjugated Polymer / Near-Field /

Many $\pi$-conjugated polymers as advanced materials for photonic and electronic applications have been developed and are the focal point of an ever-increasing interest from both academic and industrial research groups. ${ }^{1}$ These $\pi$-conjugated polymers are used as laser dyes, ${ }^{2}$ photoconductors, ${ }^{3}$ light-emitting diodes (LED), ${ }^{4}$ piezoelectric materials, ${ }^{5}$ and are have possible applications to optical data storage ${ }^{6}$ and signal processing. ${ }^{7}$ There are some uses as optical resolution membrane material. ${ }^{8-10}$

Although the $\pi$-conjugated polymer is very interesting and important material for mankind as stated above, the mechanism of the single-molecular-level of these photoand electro-functions is still not clear due to various and complicated structures of the $\pi$-conjugated system in polymer molecules. Thus, the polymers are a complex system of molecules. We theorize that if direct observation of a single molecule of the $\pi$-conjugated polymer is achieved, the relationship between polymer structure and functions should be clarified on a molecular level for a single molecule unit, that is, the "proper" molecular devices.

Recently, imaging of single protein molecules using fluorescence microscopy has made remarkable progress after the development of single fluorophore imaging methods that were used for specimens on an air-dried surface. ${ }^{11}$ Single fluorophores in aqueous solution have been imaged with a total internal reflection fluorescence microscope (TIRFM), whose background fluorescence is significantly reduced. ${ }^{12}$ When a laser beam is totally reflected on the interface between a glass surface and solution, illumination is limited to the vicinity near the glass surface.

Bout et al. reported single molecule spectra of a $\pi$ conjugated substituted poly(phenylenevinylene) in polystyrene matrix, ${ }^{13}$ but this was not a solution system. If single molecule imaging of $\pi$-conjugated polymer in solution is achieved, unique phenomena in solution can be expected, e.g., host-guest interaction of single molecules.

In this study, single fluorophore imaging was expanded to an organic polymer solution system for direct observation of single molecules of a photofunctional $\pi$ conjugated polymer. A photo-functional $\pi$-conjugated rigid-rod poly(aryleneethynylene) bearing anthracene in the main chain was synthesized by polycondensation reaction using the palladium-copper catalyst system and characterized. On the $\pi$-conjugated polymer solution TIRFM was used for detection of single molecule photoluminescence at room temperature.

\section{EXPERIMENTAL}

\section{Materials}

Triethylamine was distilled over calcium hydride. 1,4Diethynylbenzene (Tokyo Kasei), 9,10-dibromoanthracene (Tokyo Kasei) and the catalyst system ${ }^{14}$ were commercially obtained.

\section{Synthesis [Scheme 1]}

The following procedures were conducted under dry nitrogen. 1,4-Diethynylbenzene $(582 \mathrm{mg}, 4.61 \mathrm{mmol})$ and 9,10-dibromoanthracene ( $1.55 \mathrm{~g}, 4.61 \mathrm{mmol})$ were dissolved in triethylamine (TEA) (100 mL) and tetrahydrofuran (THF) $(20 \mathrm{~mL})$, followed by the addition of triphenylphosphine $(550 \mathrm{mg}, 2.10 \mathrm{mmol}$ ), cuprous iodide $(275$ $\mathrm{mg}, 1.45 \mathrm{mmol}$ ), and dichlorobis(triphenylphosphine)palladium $\left[\mathrm{PdCl}_{2}\left(\mathrm{PPh}_{3}\right)_{2}\right](298 \mathrm{mg}, 0.425 \mathrm{mmol})$. After stirring for $40 \mathrm{~h}$ at reflux temperature, the precipitated salt, triethylammonium bromide, was filtered at room temperature, and rinsed with ethyl ether. The filtrates and ether were combined and solvent was evaporated to dryness. The residue was dissolved in minimum amount of chloroform and the solution was poured into methanol $(200 \mathrm{~mL})$ to form a precipitate subsequently separated by centrifugation. The resulting polymer was dried under vacuum to give poly $(9,10$-anthracenediyl-ethynylene-1,4-phenylene-ethynylene) [Poly(AEPE)] as a red solid. Yield: $69 \%$; GPC: $M_{\mathrm{w}}=6.1 \times 10^{3}, M_{\mathrm{w}} / M_{\mathrm{n}}=3.5$ (based on polystyrene standard). UV-vis. $\lambda_{\max }=480 \mathrm{~nm}$; $\lambda_{\varepsilon<50}=550 \mathrm{~nm}$. The fluorescence spectrum of poly (AEPE) THF solution is shown in Figure 1.

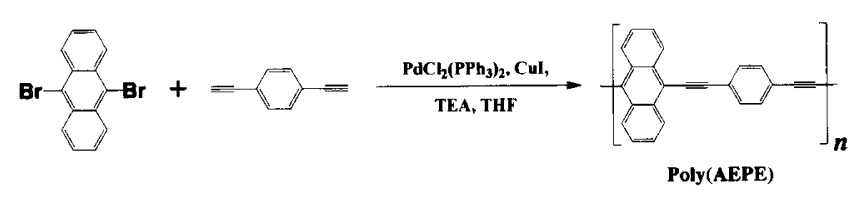

Scheme 1. Synthesis of poly $(9,10$-anthracenediyl-ethynylene1,4-phenylene-ethynylene) [Poly(AEPE)].

\footnotetext{
${ }^{\dagger}$ To whom correspondence should be addressed (Tel \& Fax:+81-22-217-7313, E-Mail: shinoken@material.tohoku.ac.jp).
} 


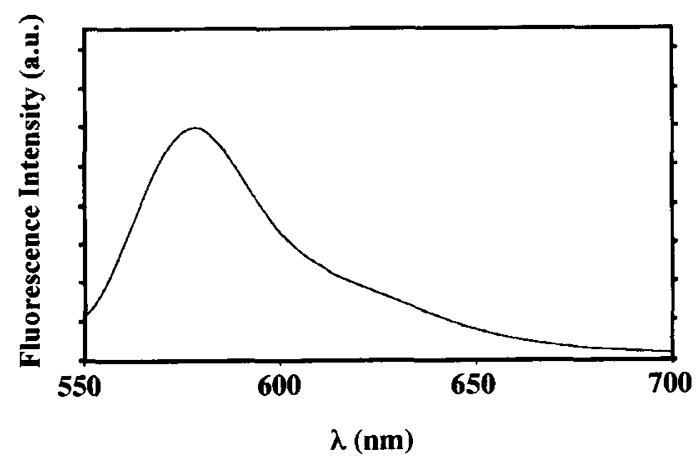

Figure 1. Fluorescence spectrum of poly(AEPE) in THF solution at r.t. Wavelength of excitation $\left(\lambda_{\text {ex }}\right): 532 \mathrm{~nm}$.
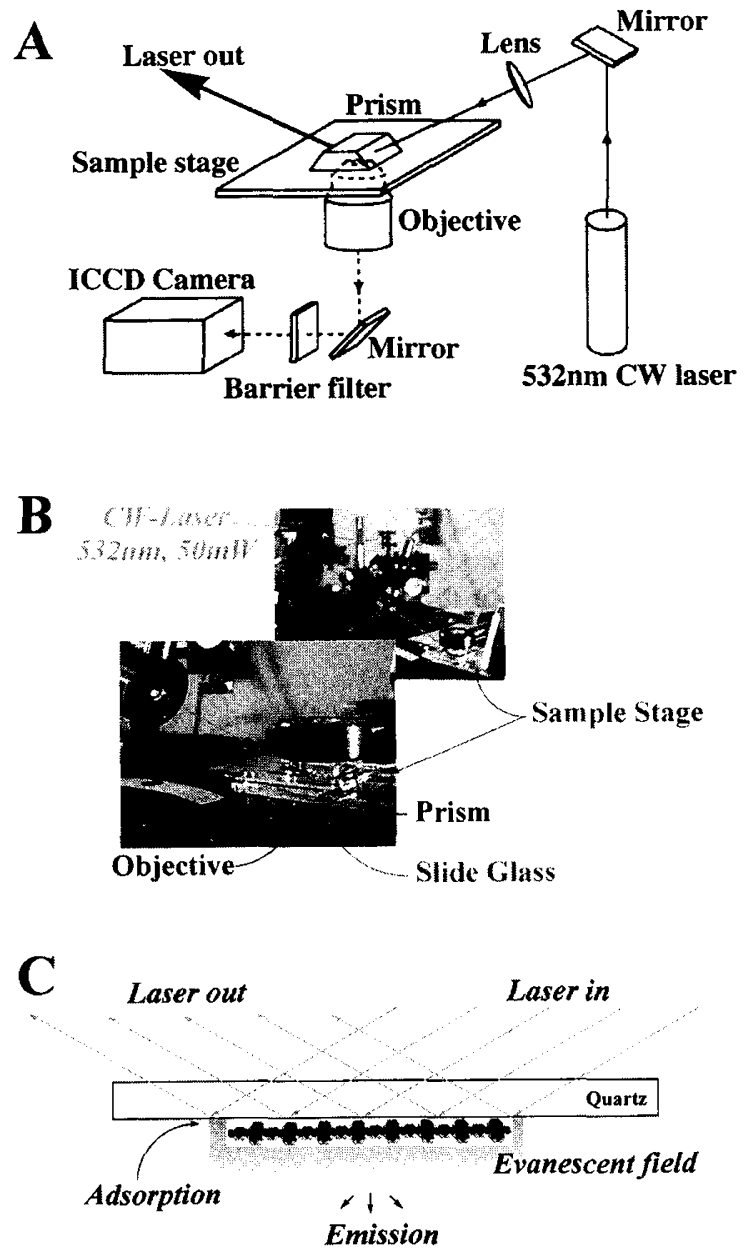

Figure 2. Schematic drawing of the optical system for single fluorophore imaging (see instrumentation of TIRFM for details)

\section{Total Internal Reflection Fluorescence Microscope (TIRFM)}

A spectroscopic device is incorporated into the TIRFM (Figure 2) based on an optical microscope (IX-70-22 FL/ PH, OLYMPUS, Japan). ${ }^{12}$ The linearly-polarized 532nm-line output of a continuous wave (CW) laser was used. After passing through a focusing lens and cubic prism, the laser beam was totally reflected. The incident angle at the quartz slide-to-solution was 78 degrees to the normal (the critical angle was 74.5 degrees). Illumination area was $200 \times 100 \mu \mathrm{m}^{2}$ at the specimen plane.
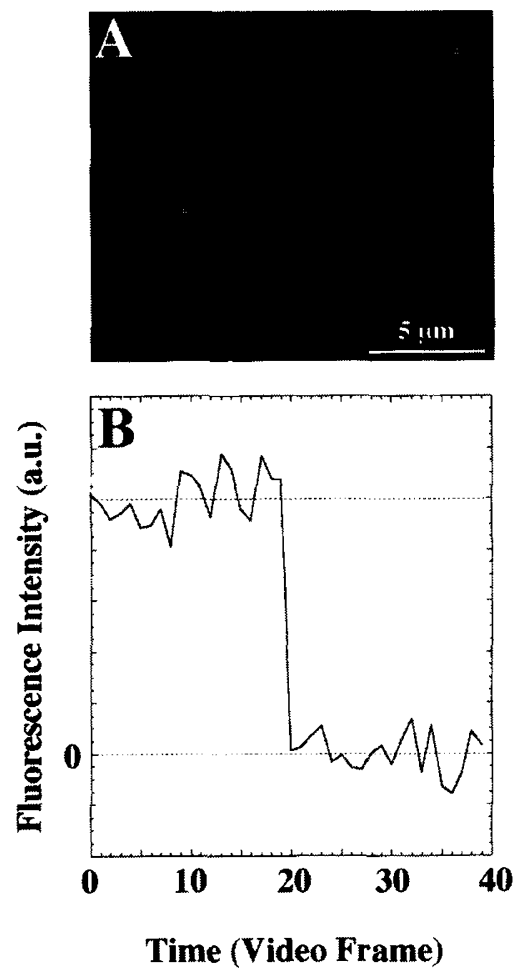

Figure 3. (A) Fluorescence image of individual single molecules of poly(AEPE) on a glass surface in THF solution. (B) Fluorescence image intensity of poly(AEPE) single molecules on glass surface in THF solution observed at video rate $(30$ frame $=1.0 \mathrm{~s})$.

Fluorescence emission from the specimen was collected with an oil-immersion microscope objective (1.40 NA, 60 $\times$, PlanApo; Nikon, Tokyo, Japan).

For imaging, fluorescence was filtered by a barrier filter (BA 580 IF; OLYMPUS, Japan) and focused by a relay lens onto an ICCD camera (ICCD 325, Photek, USA). The images were recorded on a video cassette recorder through an image processor (ARUGUS C 5510, Hamamatsu Photonics, Japan).

\section{RESULTS AND DISCUSSION}

\section{Synthesis and Characterization of Poly(AEPE)}

Using the catalyst system of bis(triphenylphosphine) palladium dichloride, cuprous iodide, and triphenylphosphine, polycondensation between the 1,4-diethynylbenzene and 9,10-dibromoanthracene (Scheme 1) gave a red solid poly(AEPE). Poly(AEPE) had an expanded $\pi$ conjugated linear main chain that absorbed a wavelength of $532 \mathrm{~nm}$ and emitted fluorescence with a peak wavelength of $578 \mathrm{~nm}$ (Figure 1) in THF. THF was a good solvent for poly(AEPE). Possible application of a TIRFM in a THF solution is described in the following.

\section{Fluorescence Images for Individual Single Poly(AEPE) Molecules}

After putting poly(AEPE) THF solution $\left(1 \times 10^{-9} \mathrm{~mol}\right.$ $\mathrm{L}^{-1}, 2 \mu \mathrm{L}$ ) on the surface of a slide glass, fluorescence images were measured with TIRFM (Figure 2). Fluorescence of a single molecule was very weak. To decrease background luminescence, we reduced the optical excitation volume using evanescent-field illumination of TIRFM. ${ }^{12}$ When a laser is incident on a quartz slide-to- 
medium interface above the critical angle, light is totally and internally reflected and an evanescent field is produced just beyond the interface. This evanescent field is localized near the interface with the $1 / e$ penetration depth of $250 \mathrm{~nm}$ depending on incident angle of the laser. Combining low background optics with local excitation, background was reduced to $1-3$ photons $\mathrm{s}^{-1}$ per diffraction limit area, i.e., $>2000$-fold lower than that for a conventional epifluorescence microscope. ${ }^{12}$ Thus, single fluorophores, that is, single poly(AEPE) molecules could be clearly visualized at a full-video rate $(1 / 30 \mathrm{~s})$ (Figure 3A). A typical time course for fluorescence intensity of an individual bright spot in the square, is shown in Figure 3B. After irradiation of the laser beam for a certain period of time, fluorescence intensity dropped to the baseline in a single-step manner due to desorption, confirming each single spot to arise from a single fluorophore.

Evidence that a single fluorescent spot is produced from a single poly(AEPE) molecule with TIRFM in THF solution was obtained as follows: 1) Poly(AEPE) easily dissolved in THF. 2) Poly(AEPE) solution was dilute (1 $\times 10^{-9} \mathrm{~mol} \mathrm{~L}^{-1}$ ). From 1) \& 2), poly(AEPE) molecules were very difficult to associate under the conditions of TIRFM measurement. 3) The fluorescent spot was desorbed in a single step (Figure 3B). If the single fluorescent spot is associated with two molecules, desorption must occur in two steps.

This is the first report that single $\pi$-conjugated polymer molecules and photofunction can be directly detected in organic solution at room temperature.

Acknowledgment. This study was partially supported by a grant-in-aid for Scientific Research on Priority Areas (A) No.286 of "Near-field Nano-optics" from the Ministry of Education, Science and Culture, Japan (No.11122204).

\section{REFERENCES}

1. R. E. Martin and F. Diederich, Angew. Chem. Int. Ed., 38, 1350 (1999).

2. H. Meier, Angew. Chem., Int. Ed., 31, 1399 (1992).

3. H. Bleier, in "Organic Materials for Photonics", Elsevier, Amsterdam, 1993, pp 77-101.

4. A. Kraft, A. C. Grimsdale, and A. B. Holmes, Angew. Chem., 110, 416 (1998).

5. D. K. Das-Gupta, in "Introduction to Molecular Electronics", Edward Arnold, London, 1995, pp 47-71.

6. L. Feringa, W. F. Jager, and B. de Lange, Tetrahedron, 49, 8267 (1993).

7. J. -M. Lehn, in "Supramolecular Chemistry. Concepts and Perspectives", VCH, Weinheim, 1995, pp 124-138.

8. K. Shinohara, T. Aoki, T. Kaneko, and E. Oikawa, Polymer, 36, 2403 (1995).

9. T. Aoki, K. Shinohara, T. Kaneko, and E. Oikawa, Macromolecules, 29, 4192 (1996).

10. a) T. Aoki, K. Shinohara, and E. Oikawa, Makromol. Chem., Rapid Commun., 13, 565 (1992). b) K. Shinohara, T. Aoki, T. Kaneko, and E. Oikawa, Chem. Lett., 361 (1997).

11. J. K. Trautman, J. J. Macklin, L. E. Brus, and E. Betzig, Nature, 369, 40 (1994).

12. a) T. Funatsu, Y. Harada, M. Tokunaga, K. Saito, and T. Yanagida, Nature, 374, 555 (1995). b) T. Funatsu, Y. Harada, H. Higuchi, M. Tokunaga, K. Saito, Y. Ishii, R. D. Vale, and T. Yanagida, Biophys. Chem., 68, 63 (1997).

13. D. V. Bout, W. -T. Yip, D. Hu, D. -K. Fu, T. M. Swager, and P. F. Barbara, Science, 277, 1074 (1997).

14. E. T. Sabourinand and A. Onopchenko, J. Org. Chem., 48, 5135 (1983). 